\title{
Investigation of mutual coupling effects in conventional and fractal capacitive coupled suspended RMSAs
}

\author{
Miti Bharatkumar Sukhadia*, Veeresh Gangappa Kasabegoudar \\ Post Graduate Department, Mahatma Basaveshwar Education Society's, College of Engineering, Ambajogai, India
}

Email address:

miti.sukhadia@yahoo.com(M. B. Sukhadia),veereshgk2002@rediffmail.com(V. G. Kasabegoudar)

\section{To cite this article:}

Miti Bharatkumar Sukhadia, Veeresh Gangappa Kasabegoudar. Investigation of Mutual Coupling Effects in Conventional and Fractal Capacitive Coupled Suspended RMSAs. International Journal of Wireless Communications and Mobile Computing, Vol. 1, No. 4, 2013, pp. 119-123. doi: 10.11648/j.wcmc.20130104.16

\begin{abstract}
An array of coplanar capacitive coupled suspended microstrip antenna has been investigated to study the mutual coupling effect between its elements. Also, the work is extended to study the mutual coupling effect in Koch shaped coplanar capacitive coupled suspended fractal microstrip antenna. From the investigations it is observed that less coupling effect occurs between the elements in fractal array. This study reveals the use of fractal geometries in place of conventional (regular) shapes because of their numerous advantages like self similarity property, ability to excite multiple resonant modes etc. Antenna arrays are simulated using IE3D software from Zeland which is based on Method of Moments (MoM).
\end{abstract}

Keywords: Microstrip Antennas, Fractal Antennas, Miniaturization, Mutual Coupling

\section{Introduction}

Due to a dynamic advancement in wireless communication industry, modern antenna components are required to have compact size, small weight, low profile, and low cost of the production [1-3]. Based on the simplicity of design and circuit implementation as well as their ability to integrate with RF devices easily, conventional microstrip patch antennas are found to be extremely useful in many wireless communication applications [4,5].

However, microstrip antennas exhibit the drawback of poor bandwidth and gain in their conventional form. There are several researches reported in literature to improve the bandwidth [6-17]. It is well known that to increase the gain of microstrip antenna, multiple elements are used. However, the spacing between the elements plays important role in calculating the area required and mutual coupling effect between the elements.

It is necessary that efficient miniaturization of $\mathrm{RF} /$ microwave antenna circuit is expected from novel mobile devices with adequate radiating properties; the solution of this limitation can be reached by means of fractal geometry. Fractal geometry occupy less space and produces same radiation patterns and input impedance as conventional geometry.

However, such radiators suffer from narrow bandwidth and insufficient radiation pattern control [6]. In order to overcome this limitation, a new antenna array is designed which allow us to achieve a modified radiation patterns as well as broadband or multiband characteristics that would finally increased the efficiency[7-10].

On the other hand, mutual coupling adversely affects the feeding voltages of the array elements, typically raising the level of the back radiation and filling in the nulls of the antenna patterns [11].

This problem can be managed by using smaller array elements, which would maintain inter-element spacing while increasing the physical gap between elements. In this paper, mutual coupling effect between the array elements is presented for conventional and fractal elements. The microstrip patch, fractal patch and array are designed and optimized. Section 2 presents the basic geometries and microstrip fractal patch with their working. Simulation results and antenna array with mutual coupling have been presented in Section 3 followed by conclusions in Section 4.

\section{Antenna Geometry}

In this section geometry description of basic element is given. The basic geometries considered are conventional coplanar capacitive coupled probe fed suspended microstrip antenna and its fractal shape. 


\subsection{Conventional Patch}

The microstrip geometry in its conventional form has limited bandwidth $(\sim 2-3 \%)$, which is inadequate for handling the high data traffic. To handle high data rates many broad banding techniques have been proposed by several researchers [10-17]. Among these a single layer coplanar capacitively coupled probe fed offers an impedance bandwidth of about $50 \%$. The geometry of this antenna is shown in Figure 1. The optimized dimensions of the geometry are presented in Table 1 [13].

Table 1. Optimized dimensions for capacitively coupled probe fed suspended patch antenna [13].

\begin{tabular}{ll}
\hline Parameter & Value \\
\hline Length of the radiator patch $(L)$ & $15.5 \mathrm{~mm}$ \\
Width of the radiator patch $(W)$ & $16.4 \mathrm{~mm}$ \\
Length of the feed strip $(s)$ & $3.7 \mathrm{~mm}$ \\
Width of the feed strip $(t)$ & $1.2 \mathrm{~mm}$ \\
Separation of feed strip from the patch $(d)$ & $0.5 \mathrm{~mm}$ \\
Air gap between substrates $(g)$ & $6.0 \mathrm{~mm}$ \\
Relative dielectric constant $(\varepsilon r)$ & 3.0 \\
\hline
\end{tabular}
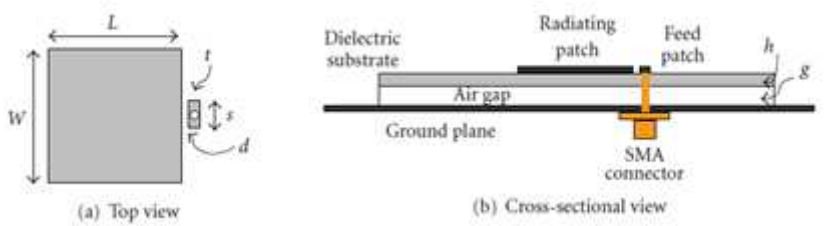

Figure 1. Coplanar capacitively coupled probe fed suspended microstrip antenna (a) Top view (b) Cross-sectional view [12].

As reported in [13], this geometry offers an impedance bandwidth of about $50 \%$ with good gain and patterns throughout the band of operation.

\subsection{Fractal Antenna Geometry}

To reduce the size of planner antenna, number of methods have been developed and reported [14-19]. It may be noted that the defects in the patch metallization lead to noticeable radiator miniaturization [14]. However, one can observe that such a technique decreases antenna resonant frequency, so antenna optimization is necessary. An alternative method of antenna size reduction is reported in [15] based on introducing a lumped element between the ground plane and signal line.

However, it is important to emphasis that such a solution adds complexity to the circuit. An integrating approach to antenna miniaturization involves the utilization of meta materials $[16,17]$. However, the use of artificial materials leads to certain fabrication problems and the design process becomes complex. One of the solutions to above limitations can be reached by means of fractal curves. It has been reported in $[18,19]$ that the application of space filling fractal curves enables us to achieve considerable miniaturization and also wideband and multi band properties.

Figure 2 shows examples of fractal geometries proposed by Koch (1904) and Sierpinski (1916).

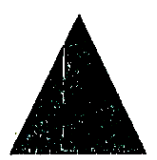

Stage 0

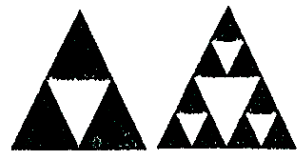

Stage $1 \quad$ Stage 2

Sierpinski gasket fractal

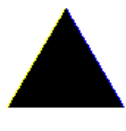

Stage 0
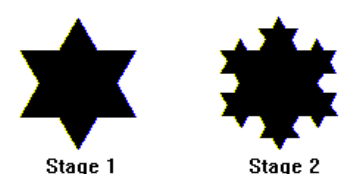

Stage 2

Koch fractal

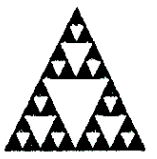

Stage 3

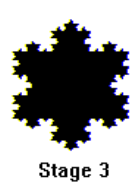

Figure 2. Typical fractal geometries [7].

Fractal has interesting properties like self-similar and space filling by iterating certain simple geometry, due to this they are use in many applications.

In this work we use the geometry presented in Figure 1 for the design of fractal antenna. This conventional antenna is treated as the base for the comparison in terms of size reduction.

Next, the structure is modified by the addition of multiple V-grooves along the length and width in three steps, which corresponds to the three iterations of the fractal generation. The addition of the groove is based upon the "Koch curve". The middle part is replaced by two straight lines meeting at 60 angles (a bent) and they fit into the original gap in an equilateral triangular fashion as shown in the Figure 3.
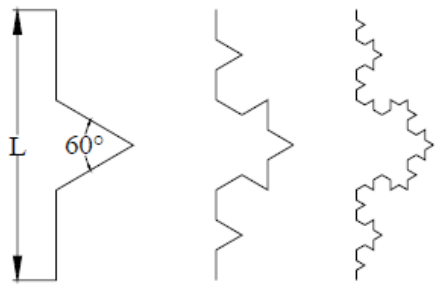

$1^{\text {st }}$ iteration $2^{\text {nd }}$ iteration $3^{\text {rd }}$ iteration

Figure 3. Fractal generation based on Koch curve [7].

Thus the dimension of each newly generated straight line is now one third of the original straight line and when each side of the square is stretched out, length increases by one third of the original length. The iterative process of dividing a straight line into three equal segments and replacing the middle by a bent curve is continued. In the true fractal, this process is repeated for infinite number of times. In the present work, three iterations are considered and the fractal patch obtained from this technique is shown in the Figure 4. 


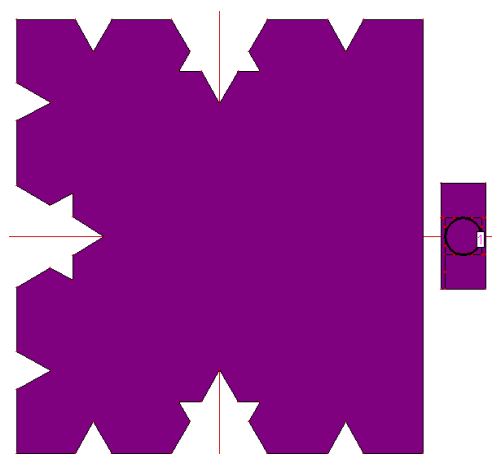

Figure 4. Coplanar capacitively coupled fractal patch.

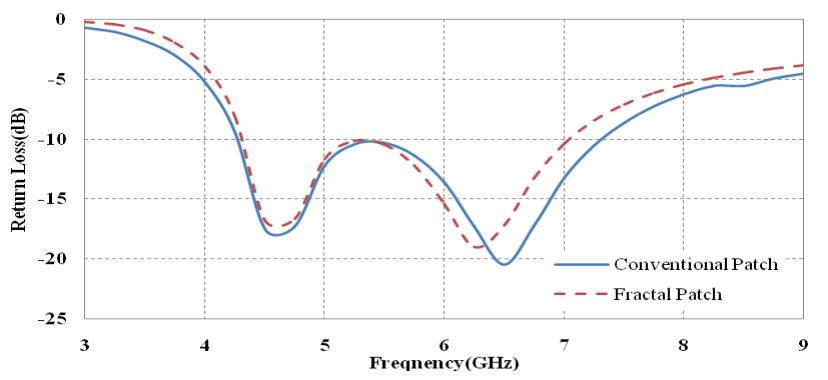

Figure 5. Return loss comparisons of fractal and conventional geometries.

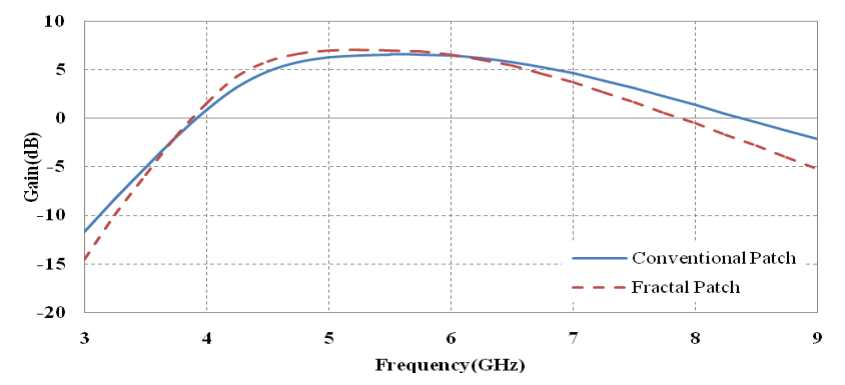

Figure 6. Gain vs. frequency plot of conventional and rectangular plots.

It is well known that microstrip antennas are good candidates for size reduction, as large electrical length can be fitted into the small physical volume. However, to make the antenna resonate at a particular frequency the useful range of size reduction lies only up to third iterations. Maximum size reduction results after first iteration only. Subsequent iterations result in decrease in percentage of size reduction such as only $5 \%$ or less than this is obtained after third iteration [7]. Therefore, one can limit the iterations up to third iterations only. Going for the higher iteration adds mostly the small edges, which is practically not useful in increasing the electrical length.

\section{Simulation Results and Discussions}

In this section two elements conventional and fractal elements arrays have been investigated to observe the coupling between the elements. All simulations have been carried out from Zeland's IE3D which is MoM based electromagnetic software. Distance between the array elements has been varied and optimized.

\subsection{Design of Two Elements Array and Effect of Mutual Coupling}

It is well known that arranging antenna elements in a predefined manner (array) can boost the performance of the radiating system like increasing the directive gain and reducing the beam width. However, the spacing between the elements plays a crucial role. Large spacing offers the enhanced gain at the expense of increased overall size and side lobes. The side lobes start appearing and become significant when spacing between the elements increases over one wavelength. Also, in applications where size is the prime constraint, increasing the spacing between the elements may not be feasible. On the other hand if the spacing is kept low results in the mutual coupling between the elements and thus disturbs the radiation characteristics and also reduces the gain of the antenna. Hence, in this work an effort has been made to find the optimum spacing between the elements of the proposed geometry and also the same is demonstrated for the two elements array with fractal geometry. Following paragraph explains the effect of mutual coupling in conventional as well as fractal arrays.

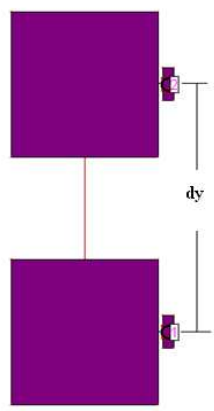

(a)

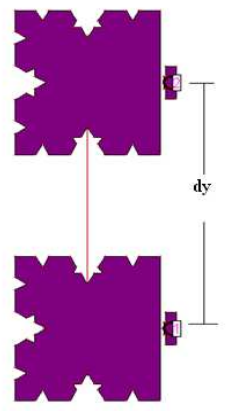

(b)
Figure 7. Two elements microstrip array placed $0.9 \lambda_{g}$ apart (a) Conventional array (b) Fractal array.

Two elements conventional as well as fractal array are shown in Figure 7. The separation between the centres of element is varied from $0.9 \lambda_{\mathrm{g}}$ to $2.25 \lambda_{\mathrm{g}}$. Where guided wavelength $\left(\lambda_{\mathrm{g}}\right)$ is calculated at the resonant frequency of $5.75 \mathrm{GHz}$. The substrate used for design and simulation is RT Duriod make laminate with relative dielectric constant of 3.0 and thickness of $1.56 \mathrm{~mm}$. For comparison, we have chosen an array of rectangular patches with same number of elements. We have assumed that all patch elements are fed uniformly (their exciting current have the same amplitude and phase). Amount of coupling between the elements of conventional and fractal arrays is presented in Figures 8 (a) and (b). It may be observed that amount of coupling is less in fractal array elements than elements in the conventional array for all cases studied. Also, similar radiation (Figure 9) and gain (Figure 10) characteristics are observed in both fractal array and conventional array. From the parametric study it is clear that if mutual coupling is main constraint, fractal arrays may be preferred. 


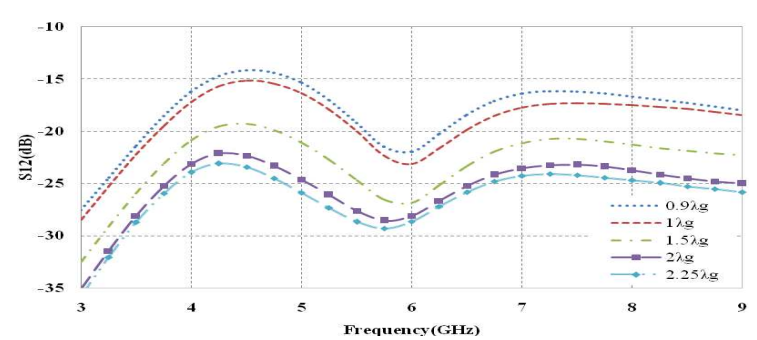

$\mathrm{S}_{12}$ plots for conventional patch elements.

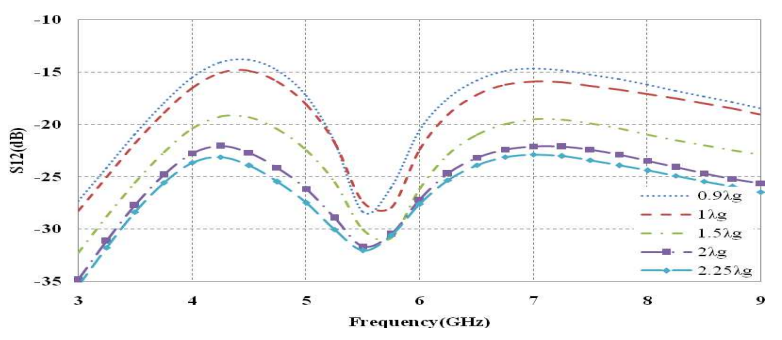

$\mathrm{S}_{12}$ plots for fractal patch elements.

Figure 8. Comparison of mutual coupling effect in antenna array elements for different spacing.

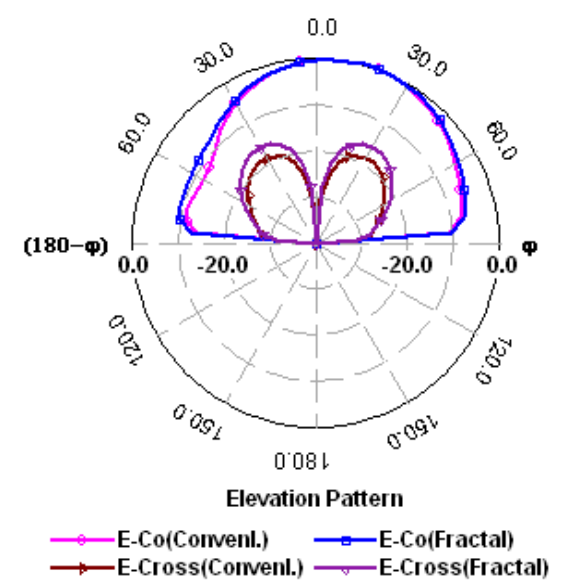

(a) E-plane patterns

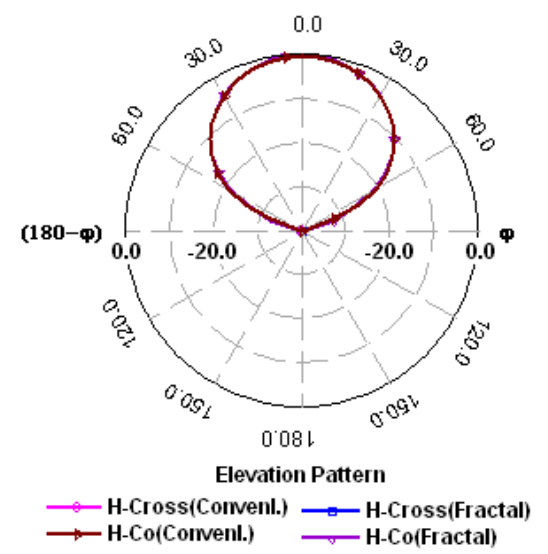

(b) H-Plane patterns

Figure 9. Comparison of radiation patterns obtained from conventional and fractal array geometries for optimum spacing between the elements $\left(0.9 \lambda_{g}\right)$ at resonant frequency.

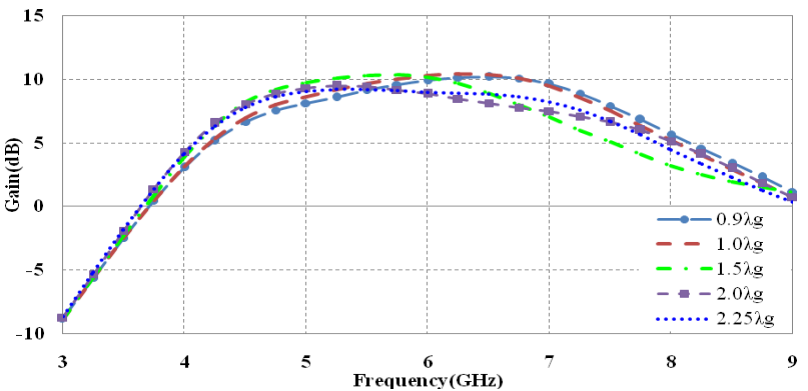

Gain vs. frequency plot for conventioanl antenna array.

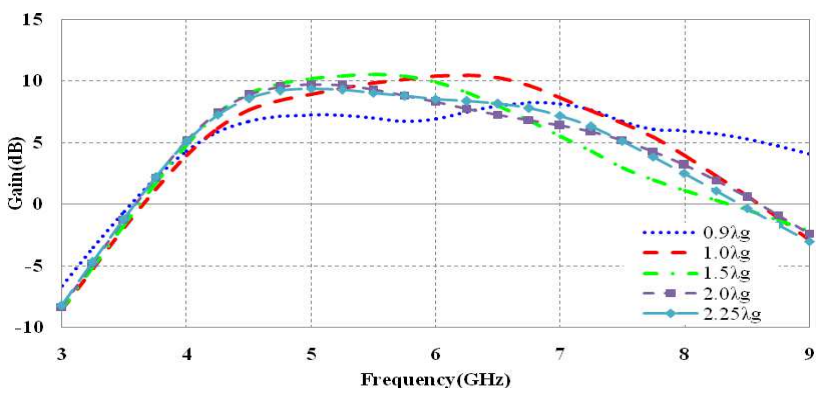

Gain vs. frequency plot for fractal antenna array.

Figure 10. Gain vs. frequency plot of antenna array elements for different spacing between the elements.

\section{Conclusions}

The fractal geometry of coplanar capacitively coupled probe fed microstrip antenna is presented. The size of antenna is reduced by increasing order of iteration. The fractal geometry offers similar performance as that of conventional patch. The effect of mutual coupling between the array elements has been presented for regular as well as fractal geometries. Fractal patches array exhibits less coupling effect between the elements with slightly increased gain. The increase in the gain is due to reduction in mutual coupling. Hence, fractal elements of capacitive coupled geometry may be used for the design of array to improve the overall performance of the antenna.

\section{References}

[1] C. A. Balanis, Antenna Theory, John Wiley \& Sons, Hoboken, NJ, USA, $2^{\text {nd }}$ Edition, 2004.

[2] R. Garg, P. Bhartia, I. Bahl, and A. Ittipiboon, Microstrip Antenna Design Handbook, Artech House, Norwood, Mass, USA, 2001.

[3] R. Kumar and P. Malathi, "On the design of fractal patch antenna and backscattering reduction," International Journal of Recent Trends In Engineering, vol. 2, pp. 4-6, 2009.

[4] J. Bahl and P. Bhartia, Microstrip Antennas, Artech House, 1981.

[5] R. Garg, "Progress in Microstrip Antennas," IETE Technical Review, vol. 18, pp. 85-98, 2001. 
[6] S. A. Fares and F. Adachi, Mobile and Wireless Communications Network Layer and Circuit Level Design, Intech, 2010.

[7] N. Yousefzadeh and C. Ghobadi, "Consideration of mutual coupling in a micro strip patch array using fractal elements," PIER, vol. 6, pp. 41-49, 2006.

[8] N. Fourikis, Advanced Array Systems, Applications and RF Technologies Phased Array Systems, Academic Press, Ascot Park, Australia, 2000.

[9] A. A. Eldek, A. Z. Elsherbeni, and C. E. Smith, "Rectangular slot antenna with patch stub for ultra wideband application and phased array systems," PIER, vol. 53, pp. 227-237, 2005 .

[10] P. S. Kooi, M. S. Leong, and T. S. Yeo, "A method of moments analysis of a micro strip hazed array in three-layered structures," PIER, vol. 31, pp. 155-179, 2001.

[11] W. L. Stutzman and G. A. Thiele, Antenna Theory and Design, John Wiley and Sons, $2^{\text {nd }}$ Edition, 1998.

[12] V. G. Kasabegoudar, "Low profile suspended microstrip antennas for wideband applications," Journal of Electromagnetic Waves and Applications, vol. 25, pp. 17951806, 2011.

[13] V. G. Kasabegoudar, D. S. Upadhyay, and K. J. Vinoy, "Design studies of ultra-wideband micro strip antennas with a small capacitive feed," International Journal of Antenna and Propagation, pp. 1-8, vol. 2007.

[14] G. A. Mavridis, C. G. Christodoulou, and M. T. Chryssomallis, "Area miniaturization of a microstrip patch antenna and the effect on the quality factor Q," IEEE Antennas and Propagation Society International Symposium, pp. 5435-5438, 2007.
[15] K. L. Wong, Compact and Broadband Microstrip Antennas, John Wiley \& Sons, 2002.

[16] R. O. Ouedraogo and E. J. Rothwell, "Metamaterial inspired patch antenna miniaturization technique," IEEE Antennas and Propagation Society International Symposium, pp. 1-4, 2010.

[17] M. Palandoken, A. Grede, and H. Henke, "Broadband microstrip antenna with left-handed metamaterials," IEEE Trans. On Antennas and Propagation, pp. 331-338, 2009.

[18] J. Guterman, A. Moreira, and C .Peixeiro, "Microstrip fractal antennas for multi standard terminals," IEEE Antennas and Wireless Propagation Letters, pp.351-354, 2004.

[19] R. V. Hariprasad, Y. Purushottam, V. C. Misra, and N. Ashok, "Microstrip fractal patch antenna for multiband communication," IEEE Electronic Letters, pp.1179-1180, 2000 .

[20] H. T. Hui and M. E. Bialkowski, "Mutual coupling in antenna arrays," International Journal of Antennas and Propagation, 2010 .

[21] C. Ludwig, "Mutual coupling, gain, and directivity of an array of two identical antennas," IEEE Transactions on Antennas and Propagation, vol. 24, no. 6, pp. 837-841, 1976.

[22] I. J. Gupta and A. A. Ksienski, "Effect of mutual coupling on the performance of adaptive arrays," IEEE Transactions on Antennas and Propagation, vol. 31, pp. 785-791, 1983.

[23] J. A. G. Malherbe, "Analysis of a linear antenna array including the effects of mutual coupling," IEEE Transactions on Education, vol. 32, pp. 29-34, 1989. 